Article

\title{
Th17/Treg Cells Imbalance and GITRL Profile in Patients with Hashimoto's Thyroiditis
}

\author{
Yingzhao Liu ${ }^{1, \dagger}$, Xinyi Tang ${ }^{2, \dagger}$, Jie Tian ${ }^{2, \dagger}$, Chenlu Zhu ${ }^{2}$, Huiyong Peng ${ }^{1}$, Ke Rui ${ }^{2}$, \\ Yungang Wang ${ }^{2}$, Chaoming Mao ${ }^{2}$, Jie Ma ${ }^{2}$, Liwei Lu ${ }^{3}$, Huaxi Xu ${ }^{2, *}$ and Shengjun Wang 1,2,*
}

1 Department of Laboratory Medicine, the Affiliated People's Hospital, Jiangsu University, Zhenjiang 212002, China; E-Mails: zjliuyingzhao@126.com (Y.L.); penghuiyong33815@163.com (H.P.)

2 Institute of Laboratory Medicine, Jiangsu Key Laboratory of Medical Science and Laboratory Medicine, Jiangsu University, Zhenjiang 212013, China; E-Mails: xinyitang0301@sina.com (X.T.); tjj850913@163.com (J.T.); zc151234@163.com (C.Z.); j827864988@163.com (K.R.); wyg1223@126.com (Y.W.); mcm20040901@126.com (C.M.); zjflmj19780723@126.com (J.M.)

3 Department of Pathology and Centre of Infection and Immunology, The University of Hong Kong, Hong Kong 999077, China; E-Mail: liweilu@hkucc.hku.hk

$\dagger$ These authors contributed equally to this work.

* Authors to whom correspondence should be addressed;

E-Mails: sjwjs@ujs.edu.cn (S.W.); xuhx@ujs.edu.cn (H.X.);

Tel.: +86-511-8503-8301 (S.W.); +86-511-8879-1048 (H.X.);

Fax: +86-511-8503-8483 (S.W. \& H.X.).

External Editor: Christophe Nicot

Received: 17 October 2014; in revised form: 7 November 2014 / Accepted: 10 November 2014 / Published: 25 November 2014

\begin{abstract}
Hashimoto's thyroiditis (HT) is an organ-specific immune disease characterized by the presence of lymphocytic infiltration and serum autoantibodies. Previous studies have confirmed the critical role of Th17 cells in the pathopoiesis of HT patients. Additionally, regulatory $\mathrm{T}$ cells (Treg) display a dysregulatory function in autoimmune disease. The purpose of this study is to investigate the alteration of Th17 and Treg cells in HT patients and explore contributing factors. We found there was an increased ratio of Th17/Treg in HT patients and a positive correlation with autoantibodies (anti-TgAb). In addition, there was an increased level of GITRL, which has been demonstrated to be correlated with the increassement of Th17 cells in the serum and thyroid glands of
\end{abstract}


HT patients; the upregulated serum level of GITRL has a positive correlation with the percentage of Th17 cells in HT patients. In summary, an increase in GITRL may impair the balance of Th17/Treg, and contribute to the pathopoiesis of Hashimoto's thyroiditis.

Keywords: Hashimoto's thyroiditis; Th17 cells; Treg cells; GITRL

\section{Introduction}

Human autoimmune thyroid disease (AITD) is an organ-specific immune disease characterized by autoantibodies, such as anti-thyroglobulin (Tg) antibody, anti-thyroperoxidase (TPO) antibody and anti-TSH receptor (TSHR) antibody, mainly involving Hashimoto's thyroiditis (HT) and Graves' disease (GD) [1-3].

$\mathrm{CD} 4^{+} \mathrm{T}$ helper $\mathrm{T}$ cells produce large amounts of cytokines in response to antigen-specific activation. They are defined as Th1, Th2, Th17 and Tfh cells on the basis of their cytokine-expression profiles. The Th17 lineage of CD4 ${ }^{+} \mathrm{T}$ helper cells produces interleukin 17 (IL-17) [4], a cytokine that induces the production of chemokines and antimicrobial peptides, leading to the recruitment of neutrophils and inflammation [5]. Th17 cells provide protection in certain infections but have also been linked to the development of autoimmune diseases, including autoimmune arthritis, Crohn's disease, multiple sclerosis, dermatomyositis and so on [6-10]. The immune response needs to be controlled to avoid an overflowing immune response, and chronic inflammation. For that goal, there is a subset of $\mathrm{CD}^{+} \mathrm{T}$ cells known to have suppressive activity and an important role in the self-tolerance, regulatory $\mathrm{T}$ cells (Treg cells) [11,12]. Th17 and Treg cells have opposite roles in the development of autoimmune diseases. While Th17 cells promote autoimmunity, Treg cells can control it and play a critical role in autoimmune pathogenesis by maintaining self-tolerance and controlling activation of autoreactive $\mathrm{CD}^{+} \mathrm{T}$ effector cells [13]. The imbalance of Th17/Treg appears also very important in the development of these diseases $[14,15]$.

Glucocorticoid-induced tumor necrosis factor receptor (GITR) family-related protein, also know as TNFRSF18, is a type 1 transmembrane protein with an extracellular $\mathrm{N}$ terminus and cytoplasmic $C$ terminus [16]. GITR is expressed at different levels in resting $\mathrm{CD}^{+}$and $\mathrm{CD}^{+} \mathrm{T}$ cells and is up-regulated after T-cell activation [17]. GITR is also constitutively expressed on $\mathrm{CD} 4^{+} \mathrm{CD} 25^{+}$Treg cells at high levels [18]. The natural ligand of GITR (GITRL) is predominantly expressed by antigen-presenting cells (APCs), including dendritic cells (DCs) and macrophages [19]. Engagement of GITR by GITRL abrogated the immunosuppressive function of Treg cells. Signaling cascades triggered through GITR and GITRL influence many physiologic and pathologic immune responses by regulating proliferation, differentiation, survival, and functions of immunocytes in both the innate and adaptive immune systems [20,21]. Additonally, an earlier study from our group has certificated a function of GITRL in exacerbating autoimmune arthritis via the enhancement of the expansion of Th17 cells [8].

It has been confirmed that the interaction of APCs, thyroidal follicular cells (TFCs) and autoreactive $\mathrm{T}$ cells results in an autoimmune response against thyroid antigens, which is mediated by Th- 1 or Th- 2 cells [22]. According to our previous studies, there was an increased frequency of Th17 cells in patients with Hashimoto's thyroiditis $[23,24]$. Apart from the enhancement of Th17 cells, there was a reduction 
of Treg cells in this study. The imbalance between Th17 cells and Treg cells may influence pathology or disease outcomes in Hashimoto's thyroiditis. We also found that the expression of GITRL was increased in HT patients, and the expression of GITRL correlated with proportions of Th17 cells.

\section{Results}

\subsection{Enhancement of Th17 Cells in Peripheral Blood from HT Patients}

Firstly, we analyzed the percentage of Th17 cells in PBMCs of HT patients by flow cytometry. Because the stimulation of PMA/ ionomycin could down-regulate the expression of human CD4 molecule, we selected $\mathrm{CD}^{+} \mathrm{CD} 8^{-}$as a marker for $\mathrm{CD} 4 \mathrm{~T}$ cells according to several previous studies $[25,26]$. We gated on $\mathrm{CD}^{+}{ }^{+} \mathrm{CD} 8^{-}$in PBMCs and identified IL- $17^{+}$cells to distinguish the Th17 cells from T cells in PBMCs (Figure 1a). It was found that HT patients showed an increase of Th17 cells at the border of statistical significance ( $p=0.056$, Figure $1 \mathrm{~b}$ ).

We next measured mRNA expression levels of ROR- $\gamma \mathrm{t}$ in PBMCs, which plays a considerable role in differentiation of Th17 cells [27]. Compared with the healthy control, HT patients had significantly higher ROR- $\gamma \mathrm{t}$ mRNA levels (Figure 1c).

It was reported that IL-6 and IL-23 are essential in the differentiation of Th17 cells [28-30]. In order to clarify the influencing factors of Th17 cells enhancement in HT patients, we analyzed the levels of IL-6 and IL-23 in serum from HT patients and healthy controls. We found that HT patients have significantly increased serum concentration of IL-6 and IL-23 in comparison with healthy controls (Figure 1d,e).

Figure 1. Enhancement of Th17 cells in peripheral blood from HT patients. PBMCs from HT patients and healthy controls were incubated with PMA/ionomycin, stained for cell surface molecules CD3 and CD8 as well as intracellular IL-17 and analyzed by flow cytometry. (a) Representative dot plots from HT patient and healthy control are shown. Values correspond to the percentage of Th17 cells. We used isotype controls to determine the positive cells, and all the values are gated on the $\mathrm{CD}^{+} \mathrm{CD}^{-}$cells; (b) Percentages of Th17 cells were compared between HT patients and healthy controls; (c) Levels of ROR- $\gamma \mathrm{t}$ mRNA in PBMCs from HT patients and healthy controls; (d) Serum levels of IL-6 were determined by ELISA in serum samples from HT patients and healthy controls and (e) Serum levels of IL-23 were determined by ELISA in serum samples from HT patients and healthy controls. $* * p<0.01 ; * * * p<0.001$.

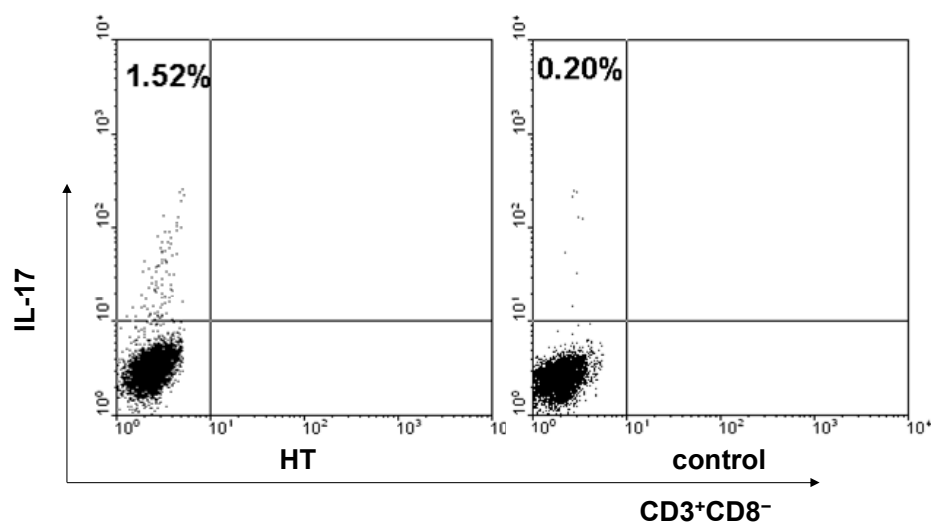

(a) 
Figure 1. Cont.

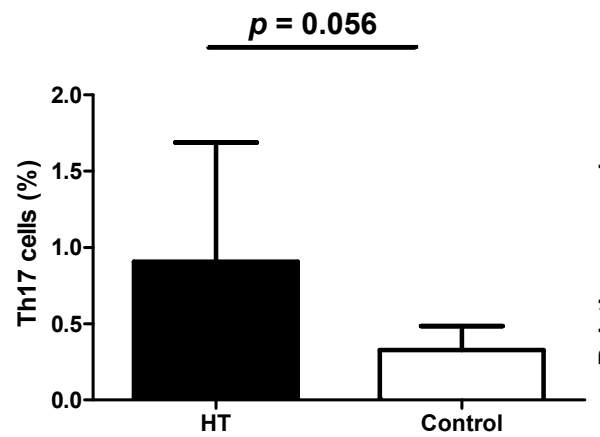

(b)

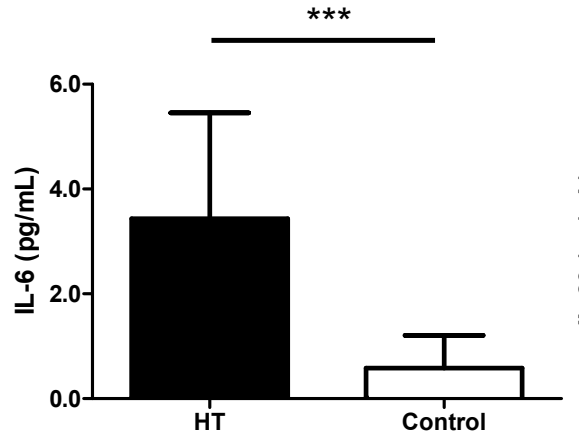

(d)

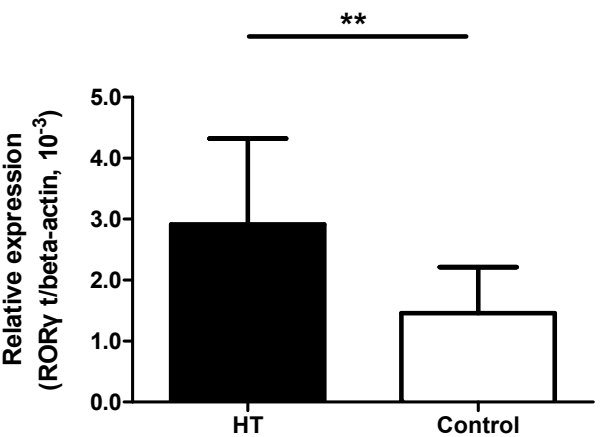

(c)

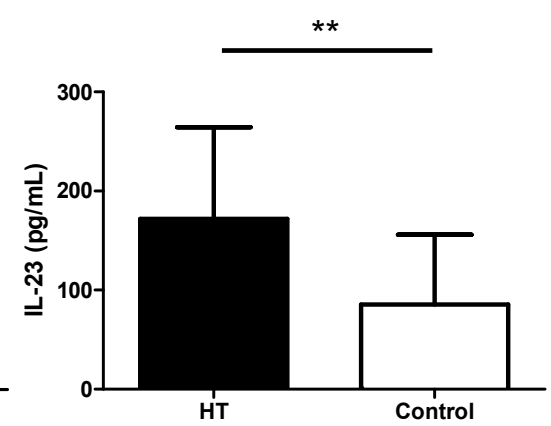

(e)

\subsection{Reduction of Regulatory T Cells in Peripheral Blood from HT Patients}

Subsequently, we gated on $\mathrm{CD} 4^{+} \mathrm{CD} 25^{+} \mathrm{CD} 127^{\text {low }} \mathrm{T}$ cells in PBMCs to distinguish Treg cells in the peripheral blood (Figure 2a). The proportion of Treg cells was reduced in PBMCs from patients with HT compared with healthy controls (Figure 2b). Foxp3 is the transcription factor of Treg cells [31]. qRT-PCR analysis displays an attenuated expression of Foxp3 mRNA in the PBMCs from HT patients (Figure 2c).

Figure 2. Reduction of regulatory $\mathrm{T}$ cells in HT patients. PBMCs from HT patients and healthy controls were stained for cell surface molecule CD4, CD25, CD127 and analyzed by flow cytometry. (a) Representative dot plots from HT patients and healthy controls are shown. Values correspond to the percentage of Treg cells. We used isotype controls to determine the positive cells, and all the values are gated on the $\mathrm{CD}^{+}$cells; (b) Percentages of Treg cells were compared between HT patients and healthy controls and (c) Levels of Foxp3 mRNA in PBMCs from HT patients and healthy controls. * $p<0.05 ; * * p<0.01$.

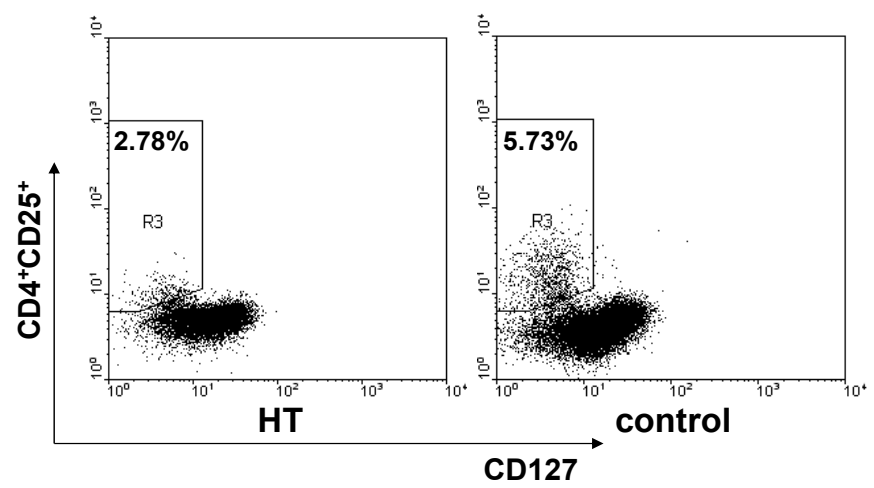

(a) 
Figure 2. Cont.

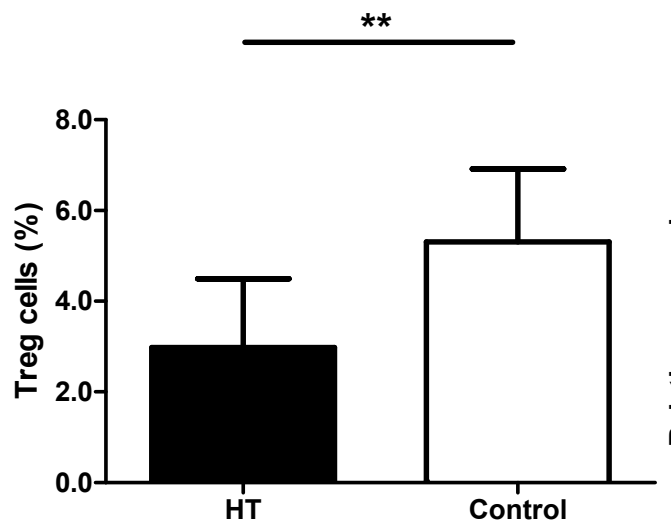

(b)

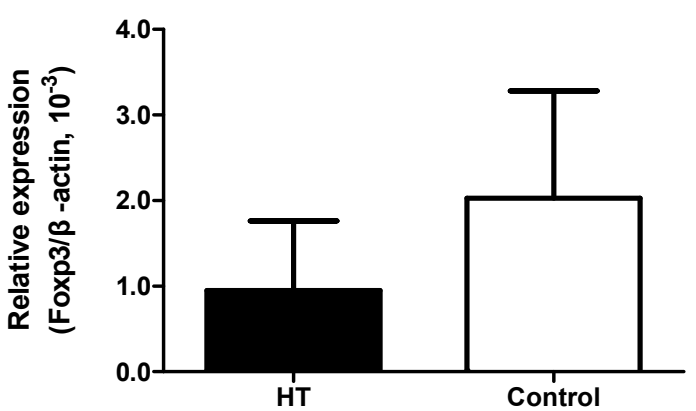

(c)

\subsection{Correlation between Th17/Treg Balance and TgAb Levels in HT Patients}

It has reported that, the control of Th17/Treg balance appears very important in the development of autoimmune diseases. We found that the ratio of Th17/Treg is significant higher in patients with HT compared with healthy controls (Figure 3a). In patients with HT, the levels of autoantibodies such as $\mathrm{TgAb}$ are upregulated, and it is one of the most important indexes of prognosis for HT. So we analyzed the correlation of TgAb levels and the ratio of Th17/Treg. By flow cytometry analysis, we found that the Th17/Treg ratio correlated positively with the TgAb levels (Figure $3 b$ ). This result demonstrated that the ratio of Th17/Treg could reflect the severity of HT in some extent.

Figure 3. Correlation between Th17/Treg balance and TgAb levels in HT patients. (a) The ratio of Th17/Treg in patients with HT compared with healthy controls and (b) The correlation between the ratio of Th17/Treg and TgAb levels in HT patients. ** $p<0.01$.

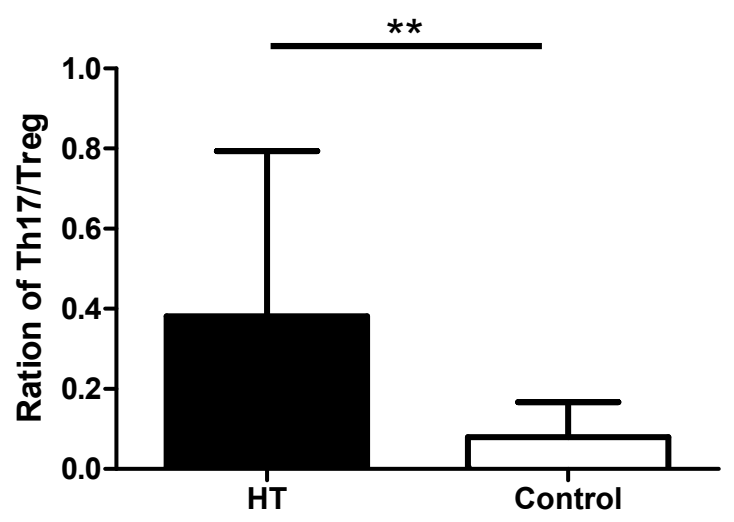

(a)

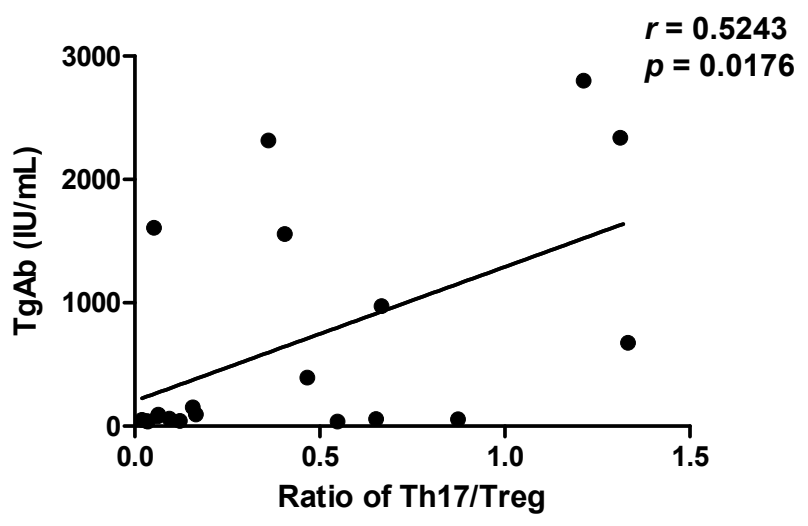

(b)

\subsection{High Levels of GITRL in Patients with HT}

Previous study has revealed that Foxp $3^{+} \mathrm{CD} 4^{+} \mathrm{CD} 25^{+} \mathrm{T}$ regulatory cell (nTreg)-mediated suppression of immune response is abrogated following combination of glucocorticoid-induced tumor necrosis receptor (GITR) family-related protein. Additionally, we have confirmed that GITRL could enhance the levels of Th17 cells in mice, and aggravate collagen-induced arthritis in mice [11]. In this study we have 
demonstrated the increased ratio of Th17/Treg in HT patients, and a positive correlation between the ratio of Th17/Treg and TgAb levels. We detected the levels of GITRL in the serum of HT patients by ELISA and found that GITRL levels are significantly augmented in the serum of HT patients as compared to healthy controls (Figure 4a). Also, there was a positive correlation between the levels of GITRL and the proportion of Th17 cells (Figure 4b). Apart from this, there is no correlation between the levels of GITRL and the percentages of Treg cells in HT patients (Figure 4c).

Figure 4. High levels of GITRL in patients with HT. (a) Serum levels of GITRL were determined by ELISA in serum samples from HT patients and controls; (b) The correlation between the percentage of Th17 cells and serum levels of GITRL in HT patients and (c) The correlation between the percentage of Treg cells and serum levels of GITRL in HT patients. $* p<0.05$.

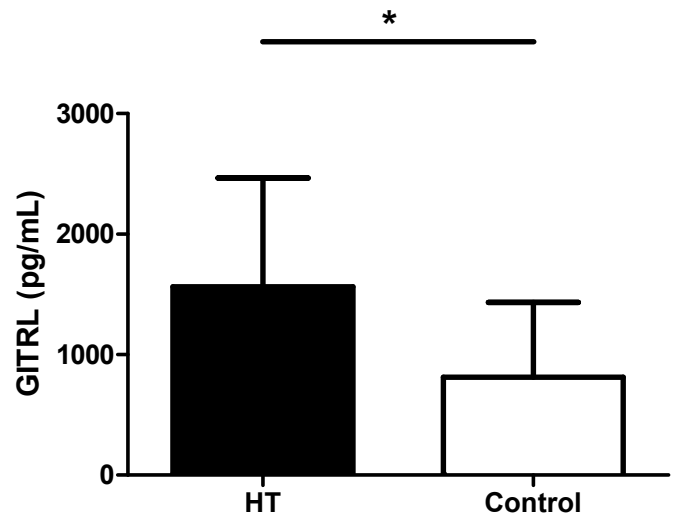

(a)

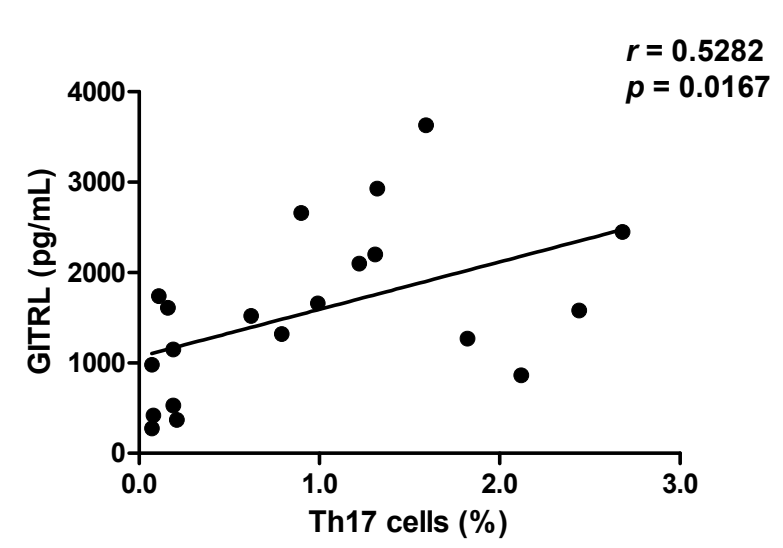

(b)

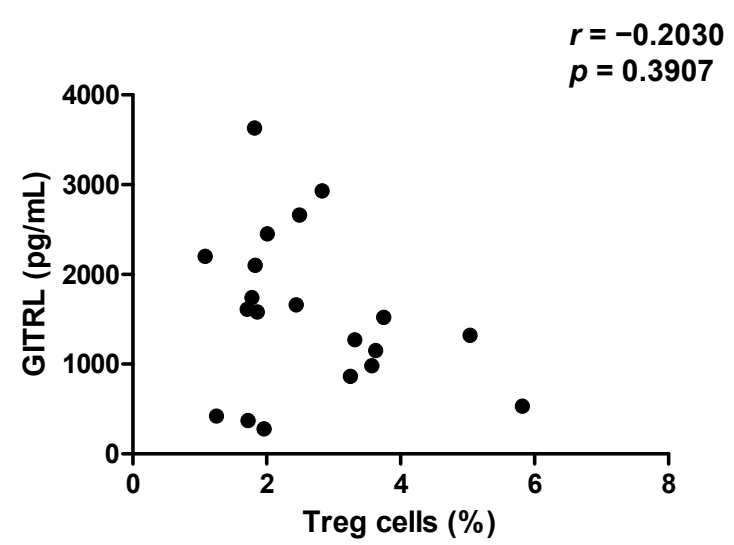

(c)

\subsection{Increased Expression of IL-17, ROR-yt and GITRL mRNA in Thyroid Tissue from HT Patients}

HT is an organ-specific immune disease, and lymphoid infiltration in thyroid tissue is a significant feature. In order to investigate the thyroid tissue, we collected six thyroid glands of HT patients. Real-time PCR showed increased expression of IL-17 and ROR- $\gamma$ t mRNA in thyroid glands from HT patients compared with simple goiter patients (Figure 5a,b). Apart from these, the expression of GITRL mRNA in the thyroid glands from HT patients was significantly higher when compared with simple goiter patients (Figure 5c). 
Figure 5. Higher expression of IL-17, ROR- $\gamma$ t and GITRL mRNA in thyroid tissue from HT patients. (a) The levels of IL-17 mRNA in thyroid glands were detected by real-time PCR from HT patients, and patients with simple goiter; (b) The levels of ROR- $\gamma \mathrm{t}$ mRNA in thyroid glands were detected by real-time PCR from HT patients, and patients with simple goiter and (c) The levels of GITRL mRNA in thyroid glands were detected by real-time PCR from HT patients, and patients with simple goiter. ${ }^{*} p<0.05$.

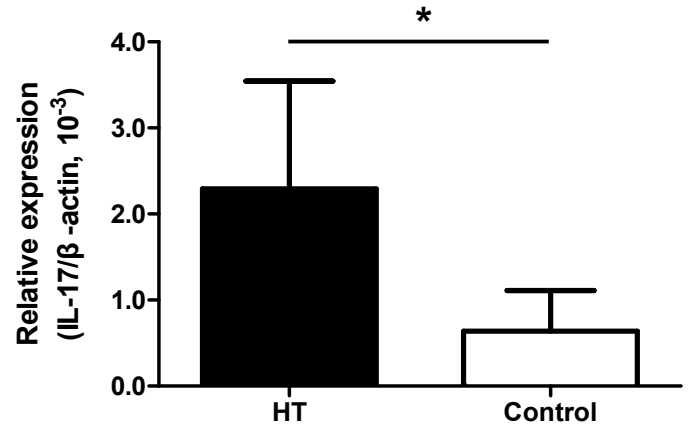

(a)

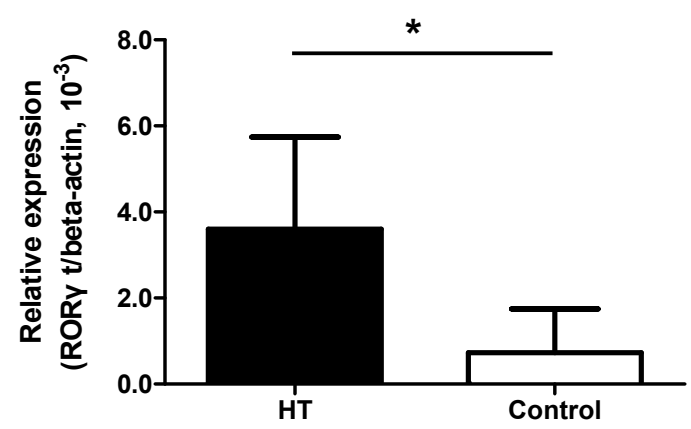

(b)

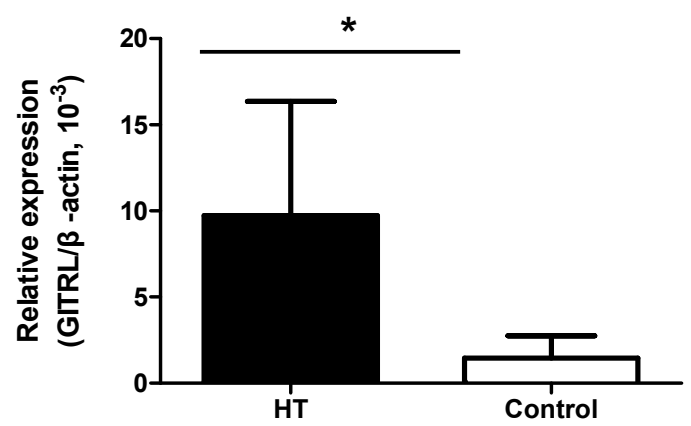

(c)

\section{Discussion}

The pathological basis of HT so far remains largely unexplored. Some studies have shown that HT is a Th1-mediated autoimmune disease. Besides Th1 cells, some other subsets of CD4 ${ }^{+} \mathrm{T}$ cells participate in the process of several autoimmune diseases, such as Th17 cells and Treg cells. These two kinds of $\mathrm{CD}^{+} \mathrm{T}$ cells have opposite roles in the development of autoimmune diseases. While Th17 cells promote autoimmunity, Treg cells can control it. This study seeks to provide insights into the involvement of Th17/Treg cells in the pathogenesis of HT.

Previous studies have found the enhancement of Th17 cells and its related cytokines in HT patients [32-35]. Apart from this, the critical function of Tregs in the process of HT have been elucidated in two recent manuscripts [36,37]. However, these studies did not clarify the balance between Th17 and Treg cells, in addition to the possible factors involved in this phenomenon. Based on this speculation, we assessed the proportion of Th17 and Treg cells in the peripheral blood of 25 patients with HT and 20 healthy controls. We identified $\mathrm{CD}^{+} \mathrm{CD} 8^{-} \mathrm{IL}-17^{+}$cells to distinguish the Th17 cells from PBMCs. Increased Th17 cells were found in PBMCs from patients with HT. The expression of IL-17 mRNA was upregulated in the thyroid glands of HT patients. And there was an increased expression of ROR- $\gamma \mathrm{t}$ mRNA in PBMCs as well as in the thyroid glands of HT patients. The levels of IL-6 and IL-23, which play an important role in the differentiation of Th17, were increased in HT 
patients' serum. Apart from this, we identified $\mathrm{CD} 4^{+} \mathrm{CD} 25^{+} \mathrm{CD} 127^{\text {low }}$ cells to distinguish Treg cells from PBMCs. As expected, the percentage of $\mathrm{CD} 4^{+} \mathrm{CD} 25^{+} \mathrm{CD} 127^{\text {low }}$ cells was decreased in PBMCs of HT patients. The expression of Foxp3 mRNA in PBMCs from HT patients was also attenuated. These data confirmed the important role of Th17 and Treg cells in the pathogenesis of HT. In addition to this, the ratio of Th17/Treg was significantly higher in HT patients, indicating that the imbalance of Th17/Treg may be involved in the development of HT.

It is widely accepted that the upregulation of autoantibodies such as $\operatorname{TgAb}$ is the most objective clinical manifestations of HT; it could indicate the development of HT and prognosis for this disease. We found an increasing ratio of Th17/Treg in HT patients in the present study. We thus analyzed the correlation of TgAb levels and the high ratio of Th17/Treg. A positive correlation was found between $\mathrm{TgAb}$ levels and the ratio of Th17/Treg. These results collectively indicate that the imbalance of Th17/Treg could reflect the severity of HT to some extent.

GITR is a type 1 transmembrane protein induced on T cells following TCR stimulation or by the glucocorticoid dexamethasone [16,38]. It is expressed at different levels in $\mathrm{CD} 4^{+}$and $\mathrm{CD} 8^{+} \mathrm{T}$ cells, $\mathrm{CD} 4^{+} \mathrm{CD} 25^{+}$Tregs constitutively express high levels of GITR on the cell surface $[17,18]$. The natural ligand of GITR, GITRL, is predominantly expressed by antigen-presenting cells [19]. It has been reported that the engagement of GITR by GITRL abrogated the immunosuppressive function of Treg cells [18]. GITRL could also reinforce autoimmune arthritis via the enhancement of the expansion of Th17 cells [8]. To further analyze the imbalance of Th17/Treg in HT patients, we detected the levels of GITRL in the serum of HT patients and the expression of GITRL mRNA in the thyroid glands from HT patients. The levels of GITRL were significantly increased in the serum of HT patients as compared to healthy controls, and the expression of GITRL mRNA in the thyroid glands from HT patients was significantly higher when compared with patients with simple goiter either. Also, there was a positive correlation between the levels of GITRL and the proportion of Th17 cells. But there is no correlation between the levels of GITRL and the percentages of Treg cells in HT patients. According to recent studies, the $\mathrm{CD} 4{ }^{+} \mathrm{CD} 25^{\text {low }} \mathrm{GITR}{ }^{+}$cell is a novel human $\mathrm{CD} 4^{+} \mathrm{T}$-cell population with regulatory activity and expanded in patients with Sjögren's syndrome or SLE [39-41]; the results of these studies provide new insight for our follow-up study.

In summary, our data suggest that increases in Th17 cells may be caused by increased levels of GITRL, and that the high ratio of Th17/Treg could participate in the pathopoiesis of Hashimoto's thyroiditis.

\section{Experimental Section}

\subsection{Individuals and Samples}

Twenty-five patients with Hashimoto's thyroiditis were included in this study. The diagnosis was based on commonly accepted classification criteria. All the patients were on the period of onset of HT at the time of this study. Main clinical data of these patients are shown in Table 1. Peripheral blood samples were obtained from all patients. The serum concentrations of anti-TgAb and TPO-Ab were detected by chemiluminescent immunoassay (MAGLUMI 2000 PLUS, Shenzhen New Industries Biomedical Engineering Co., Shenzhen, China). All samples were taken in accordance with the regulations and approval of the Affiliated People's Hospital of Jiangsu University. 
Table 1. Clinical features of HT patients and healthy controls enrolled in the study.

\begin{tabular}{cccc}
\hline & HT Patients & Healthy Controls & Range \\
\hline Number & 25 & 20 & \\
Gender (M/F) & $5 / 20$ & $3 / 17$ & \\
Age (year) & $47.2 \pm 9.9$ & $46.3 \pm 7.2$ & \\
Tg-Ab (IU/mL) & $677.1 \pm 312.3$ & $19.4 \pm 9.3$ & $<30$ \\
TPO-Ab (IU/mL) & $317.5 \pm 206.7$ & $16.6 \pm 7.1$ & $<10$ \\
\hline
\end{tabular}

Data correspond to the arithmetic mean $\pm \mathrm{SD}$.

\subsection{Cell Isolation and Stimulation in Vitro}

Peripheral blood mononuclear cells (PBMCs) were isolated by density-gradient centrifugation over Ficoll-Hypaque solution. After isolation PBMCs were incubated in complete RPMI 1640 culture medium in the presence of $1.0 \mu \mathrm{g} / \mathrm{mL}$ ionomycin and $50 \mathrm{ng} / \mathrm{mL}$ phorbol myristate acetate (PMA; Sigma-Aldrich, St. Louis, MO, USA) for $5 \mathrm{~h}$. After $5 \mathrm{~h}$ of culture at $37{ }^{\circ} \mathrm{C}$ under $5 \% \mathrm{CO}_{2}$, cells were collected and centrifuged for Th17 cells and mRNA detection by flow cytometric analysis and qRT-PCR, respectively. Unstimulated PBMCs were used for the staining of Treg cells.

Thyroid specimens were minced and then digested with collagenase II (Sigma-Aldrich, St. Louis, MO, USA) for $1-2 \mathrm{~h}$ at $37{ }^{\circ} \mathrm{C}$, then these cells were isolated by density-gradient centrifugation over Ficoll-Hypaque solution. Thyroid mononuclear cells (TMC) were obtained and cell viability was more than $95 \%$.

\subsection{Flow Cytometric Analysis}

Unstimulated PBMCs were washed and immunostained with fluorescein isothiocyanate-conjugated anti-CD4 (eBioscience, San Diego, CA, USA), phycoerythrin (PE)-conjugated anti-CD25 (eBioscience, San Diego, CA, USA), phycoerythrin-cy5-conjugated anti-CD127 (eBioscience, San Diego, CA, USA) mAb against human cells surface. Stimulated PBMCs were immunostained with phycoerythrin-cy5-conjugated anti-CD3 (eBioscience, San Diego, CA, USA), fluorescein isothiocyanate-conjugated anti-CD8 (eBioscience, San Diego, CA, USA), phycoerythrin (PE)-conjugated anti-IL-17 (eBioscience, San Diego, CA, USA) mAb against human cells. All the staining was according to manufacturers' protocol. Isotype-matched Ab controls were used in all procedures. Cells were analyzed using a FACSCalibur flow cytometer and CELLQUEST software (Becton Dickinson, Sparks, MD, USA). The results were expressed as the percentage of $\mathrm{CD} 4^{+} \mathrm{CD} 25^{+} \mathrm{CD} 127^{\text {low }}$ cells and $\mathrm{CD} 3^{+} \mathrm{CD} 8^{-}$cells expressing IL- 17 .

\subsection{RNA Isolation and Real-Time PCR}

For the detection of cytokine IL-17, transcription factor RORC and Foxp3, PBMCs were stimulated for $5 \mathrm{~h}$ as described above. After that activated cells were used to quantify the expression of IL-17, ROR $\gamma t$ and Foxp3mRNA by real-time PCR. TRIzol reagent (Invitrogen, Carlsbad, CA, USA) was added in stimulated PBMCs. After isolated total RNA, reverse transcription was performed according to the manufacturer's instruction (Toyobo, Osaka, Japan). Real-time PCR was performed in duplicate using Bio-Rad SYBRGreen super mix (Bio-Rad, Hercules, CA, USA). Primer sequences were as follows: 
IL-17, sense, 5'-CAAGACTGAACACCGACTAAG-3'; antisense, 5'-TCTCCAAAGGAAGCCTGA-3', ROR $\gamma$ t, sense, 5'-CCTGGGCTCCTCGCCTGACC-3'; antisense, 5'-TCTCTCTGCCCTCAGCCTTGCC-3', Foxp3, sense, 5'-ACAGCACATTCCCAGAGTTCCT-3'; antisense, 5'-Rev:TCTCCACCCGCAC AAAGCA-3'.

We used $\beta$-actin as internal control. Data were analyzed by Bio-Rad CFX Manager software.

\subsection{Cytokine Quantification}

Levels of IL-6, IL-23 (eBioscience, San Diego, CA, USA) and GITRL (R\&D Systems, Inc., Minneapolis, MN, USA) were determined by ELISA method using an ELISA reader ( $\mu$ Quant, BIO-TEK, Winooski, VT, USA). All determinations were performed by duplicate and the lower detection limits for IL-6, IL-23 and GITRL were 1.5625, 15.625 and $125 \mathrm{pg} / \mathrm{mL}$ respectively.

\subsection{Statistical Analysis}

Student's unpaired $t$-test was performed to determine whether there was a statistically significant change between two groups. Correlations between variables were determined by Spearman's correlation coefficient. Data were analyzed with GraphPad Prism5 software (GraphPad Software, Inc., San Diego, CA, USA).

\section{Acknowledgments}

This study was supported by National Natural Science Foundation of China (Grant No. 30871193, 81072453, 31100648, 81310108020), Specialized Research Fund for the Doctoral Program of Higher School (Grant No. 20133227110008), Specialized Project for Clinical Medicine of Jiangsu Province (Grant No. BL2014065), Health Department Foundation of Jiangsu Province (Grant No.Z201312), Science and technology support program (Social Development) of Zhenjiang (Grant No. SH2014039), Graduate Student Research and Innovation Program of Jiangsu Province (Grant No. CXZZ13_0700, KYLX_1074), Jiangsu Province “333” Project and "Qinglan” Project.

\section{Author Contributions}

Conceived and designed the experiments: Shengjun Wang, Huaxi Xu, Yingzhao Liu. Performed the experiments: Yingzhao Liu, Xinyi Tang, Chenlu Zhu, Jie Tian. Analyzed the data: Yingzhao Liu, Xinyi Tang, Jie Tian, Chenlu Zhu, Huiyong Peng, Jie Ma, Liwei Lu. Contributed reagents/materials/ analysis tools: Ke Rui, Yungang Wang, Chaoming Mao. Wrote the paper: Yingzhao Liu, Xinyi Tang, Jie Tian, Shengjun Wang.

\section{Abbreviations}

GITRL: glucocorticoid-induced TNF receptor family-related protein ligand; HT: Hashimoto's thyroiditis. 


\section{Conflicts of Interest}

The authors declare no conflict of interest.

\section{References}

1. Pearce, E.N.; Farwell, A.P.; Braverman, L.E. Thyroiditis. N. Engl. J. Med. 2003, 348, 2646-2655.

2. Weetman, A.P. Autoimmune thyroid disease. Autoimmunity 2004, 37, 337-340.

3. Zhu, C.; Ma, J.; Liu, Y.; Tong, J.; Tian, J.; Chen, J.; Tang, X.; Xu, H.; Lu, L.; Wang, S. Increased frequency of follicular helper $\mathrm{T}$ cells in patients with autoimmune thyroid disease. J. Clin. Endocrinol. Metab. 2012, 97, 943-950.

4. Park, H. A distinct lineage of cd4 t cells regulates tissue inflammation by producing interleukin 17. Nat. Immunol. 2005, 6, 1133-1141.

5. Weaver, C.T.; Hatton, R.D.; Mangan, P.R.; Harrington, L.E. Il-17 family cytokines and the expanding diversity of effector t cell lineages. Annu. Rev. Immunol. 2007, 25, 821-852.

6. Cascão, R.; Moura, R.A.; Perpétuo, I.; Canhão, H.; Sousa, E.V.; Mourão, A.F.; Rodrigues, A.M.; Pereira, J.P.; Queiroz, M.V.; Rosário H.S. Identification of a cytokine network sustaining neutrophil and th17 activation in untreated early rheumatoid arthritis. Arthritis Res. Ther. 2010, 12, 196-204.

7. Seiderer, J.; Elben, I.; Diegelmann, J.; Glas, J.; Stallhofer, J.; Tillack, C.; Pfennig, S.; Jürgens, M.; Schmechel, S.; Konrad, A.; et al. Role of the novel Th17 cytokineIL-17F in inflammatory bowel disease (IBD): Upregulated colonic IL-17F expression in active crohn's disease and analysis of the IL17F p.His161Arg polymorphism in IBD. Inflamm. Bowel Dis. 2008, 14, 437-445.

8. Wang, S.; Shi, Y.; Yang, M.; Ma, J.; Tian, J.; Chen, J.; Mao, C.; Jiao, Z.; Ko, K.-H.; Baidoo, S.E.; et al. Gitrl exacerbates collagen-induced arthritis via enhancing the expansion of Th17 cells. Am. J. Pathol. 2012, 180, 1059-1067.

9. Tzartos, J.S.; Manuel, A.F.; Craner, M.J.; Palace, J.; Newcombe, J.; Esiri, M.M.; Fugger, L. Interleukin-17 production in central nervous system infltrating $\mathrm{T}$ cells and glial cells is associated with active disease in multiple sclerosis. Am. J. Pathol. 2008, 172, 146-155.

10. Tang, X.; Tian, X.; Zhang, Y.; Wu, W.; Tian, J.; Rui, K.; Tong, J.; Lu, L.; Xu, H.; Wang, S. Correlation between the frequency of Th17 cell and the expression of microrna-206 in patients with dermatomyositis. Clin. Dev. Immunol. 2013, 2013, 7.

11. Sakaguchi, S. Naturally arising $\mathrm{CD}^{+}$regulatory $\mathrm{T}$ cells for immunologic self-tolerance and negative control of immune responses. Annu. Rev. Immunol. 2004, 22, 531-562.

12. Wan, Y.Y.; Flavell, R.A. Tgf-beta and regulatory $\mathrm{T}$ cell in immunity and autoimmunity. J. Clin. Immunol. 2008, 28, 647-659.

13. Wang, S.; Xu, H.; Wang, Y.; Ma, J.; Mao, C.; Shao, Q.; Ma, B.; Xu, W.; Yang, S. Regulatory T cells induced by rAAV carrying the forkhead box $\mathrm{p} 3$ gene prevent autoimmune thyroiditis in mice. Int. J. Mol. Med. 2006, 18, 1193-1199.

14. Bettelli, E.; Carrire, Y.; Gao, W.; Korn, T.; Strom, T.B.; Oukka, M.; Weiner, H.L.; Kuchroo, V.K. Reciprocal developmental pathways for the generation of pathogenic effector Th17 and regulatory T cells. Nature 2006, 441, 235-238. 
15. Dieckmann, D; Plottner, H.; Berchtold, S.; Berger, T.; Schuler, G. Ex vivo isolation and characterization of $\mathrm{CD}^{+} \mathrm{CD} 25^{+} \mathrm{T}$ cells with regulatory properties from human blood. J. Exp. Med. 2001, 193, 1303-1310.

16. Kwon, B.; Yu, K.Y.; Ni, J.; Yu, G.L.; Jang, I.K.; Kim, Y.J.; Xing, L.; Liu, D.;Wang, S.X.; Kwon, B.S. Identification of a novel activation-inducible protein of the tumor necrosis factor receptor superfamily and its ligand. J. Biol. Chem. 1999, 274, 6056-6061.

17. Kim, B.J.; Zhu, L.; Fariss, R.N.; Shen, D.F.; Mahesh, S.P.; Egwuagu, C.; Yu, C.-R.; Nagineni, C.N.; Chan, C.-C.; Nussenblatt, R.B. Constitutive and cytokine-induced GITR ligand expression on human retinal pigment epithelium and photoreceptors. Investig. Ophthalmol. Vis. Sci. 2004, 45, 3170-3176.

18. McHugh, R.S.; Whitters, M.; Piccirillo, C.A.; Young, D.A.; Shevach E.M.; Collins, M.; Byrne, M.C. $\mathrm{CD} 4{ }^{+} \mathrm{CD} 25^{+}$immunoregulatory $\mathrm{T}$ cells: Gene expression analysis reveals a functional role for the glucocorticoid-induced tnf receptor. Immunity 2002, 16, 311-323.

19. Kamimura, Y.; Iwai, H.; Piao, J.; Hashiguchi, M.; Azuma, M. The glucocorticoid-induced TNF receptor-related protein (GITR)-GITR ligand pathway acts as a mediator of cutaneous dendritic cell migration and promotes $\mathrm{T}$ cell-mediated acquired immunity. J. Immunol. 2009, 182, 2708-2716.

20. Nocentini, G.; Ronchetti, S.; Petrillo, M.G.; Riccardi, C. Pharmacological modulation of GITRL/GITR system: Therapeutic perspectives. Br. J. Pharmacol. 2012, 165, 2089-2099.

21. Azuma, M. Role of the glucocorticoid-induced tnfr-related protein (GITR)-GITR ligand pathway in innate and adaptive immunity. Crit. Rev. Immunol. 2010, 30, 547-557.

22. Santaguida, M.G.; Nardo, S.; del Duca, S.C.; Lococo, E.; Virili, C.; Gargano, L.; Lenti, L.; Centanni, M. Increased interleukin-4-positive lymphocytes in patients with hashimoto's thyroiditis and concurrent non-endocrine autoimmune disorders. Clin. Exp. Immunol. 2011, 165, 148-154.

23. Wang, S.; Baidoo, S.E.; Liu, Y.; Zhu, C; Tian, J.; Ma, J.; Tong, J.; Chen, J.; Tang, X.; Xu, H.; et al. $\mathrm{T}$ cell-derived leptin contributes to increased frequency of Th17 cells in female patients with hashimoto's thyroiditis. Clin. Exp. Immunol. 2013, 171, 63-68.

24. Shi, Y.; Wang, H.; Su, Z.; Chen, J.; Xue, Y.; Wang, S.; He, Z.; Yang, H.; Zhou, C.; Kong, F.; et al. Differentiation imbalance of Th1/Th17 in peripheral blood mononuclear cells might contribute to pathogenesis of hashimoto's thyroiditis. Scand. J. Immunol. 2010, 72, 250-255.

25. Baran, J.; Kowalczyk, D.; Ozog, M.; Zembala, M. Three-color flow cytometry detection of intracellular cytokines in peripheral blood mononuclear cells: Comparative analysis of phorbol myristate acetate-ionomycin and phytohemagglutinin stimulation. Clin. Diagn. Lab. Immunol. 2001, 8, 303-313.

26. Faas, M.M.; Bouman, A.; Veenstra van Nieuwenhoven, A.L.; van der Schaaf, G.; Moes, H.; Heineman, M.J.; de Vos, P. Species differences in the effect of pregnancy on lymphocyte cytokine production between human and rat. J. Leukoc. Biol. 2005, 78, 946-953.

27. Ivanov, I.I.; McKenzie, B.S.; Zhou, L.; Tadokoro, C.E.; Lepelley, A.; Lafaille, J.J.; Cua, D.J.; Littman, D.R. The orphan nuclear receptor rorgammat directs the differentiation program of proinflammatory IL-17 ${ }^{+}$T helper cells. Cell 2006, 126, 1121-1133.

28. Volpe, E.; Servant, N.; Zollinger, R.; Bogiatzi, S.I.; Hupe, P.; Barillot, E.; Soumelis, V. A critical function for transforming growth factor-beta, interleukin 23 and proinflammatory cytokines in driving and modulating human T(h)-17 responses. Nat. Immunol. 2008, 9, 650-657. 
29. Acosta-Rodriguez, E.V.; Napolitani, G.; Lanzavecchia, A.; Sallusto, F. Interleukins 1 beta and 6 but not transforming growth factor-beta are essential for the differentiation of interleukin 17-producing human t helper cells. Nat. Immunol. 2007, 8, 942-949.

30. Annunziato, F.; Cosmi, L.; Liotta, F.; Maggi, E.; Romagnani, S. Main features of human T helper 17 cells. Ann. N. Y. Acad. Sci. 2013, 1284, 66-70.

31. Fontenot, J.D.; Gavin, M.A.; Rudensky, A.Y. Foxp3 programs the development and function of $\mathrm{CD} 4{ }^{+} \mathrm{CD} 25^{+}$regulatory t cells. Nat. Immunol. 2003, 4, 330-336.

32. Ruggeri, R.M.; Saitta, S.; Cristani, M.; Giovinazzo, S.; Tigano, V.; Trimarchi, F.; Benvenga, S.; Gangemi, S. Serum interleukin-23 (IL-23) is increased in hashimoto's thyroiditis. Endocr. J. 2014, 61, 359-363.

33. Qin, Q.; Liu, P.; Liu, L.; Wang, R.; Yan, N.; Yang, J.; Wang, X.; Pandey, M.; Zhang, J.A. The increased but non-predominant expression of Th17- and Th1-specific cytokines in hashimoto's thyroiditis but not in graves' disease. Braz. J. Med. Biol. Res. 2012, 45, 1202-1208.

34. Li, D.; Cai, W.; Gu, R.; Zhang, Y.; Zhang, H.; Tang, K.; Xu, P.; Katirai, F.; Shi, W.; Wang, L.; et al. Th17 cell plays a role in the pathogenesis of hashimoto's thyroiditis in patients. Clin. Immunol. 2013, 149, 411-420.

35. Figueroa-Vega, N.; Alfonso-Perez, M.; Benedicto, I.; Sanchez-Madrid, F.; Gonzalez-Amaro, R.; Marazuela, M. Increased circulating pro-inflammatory cytokines and Th17 lymphocytes in hashimoto's thyroiditis. J. Clin. Endocrinol. Metab. 2010, 95, 953-962.

36. Glick, A.B.; Wodzinski, A.; Fu, P.; Levine, A.D.; Wald, D.N. Impairment of regulatory T-cell function in autoimmune thyroid disease. Thyroid 2013, 23, 871-878.

37. Bossowski, A.; Moniuszko, M.; Dabrowska, M.; Sawicka, B.; Rusak, M.; Jeznach, M.; Wojtowicz, J.; Bodzenta-Lukaszyk, A.; Bossowska, A. Lower proportions of $\mathrm{CD}^{+} \mathrm{CD} 25^{\text {high }}$ and $\mathrm{CD} 4{ }^{+} \mathrm{Foxp} 3$, but not $\mathrm{CD} 4^{+} \mathrm{CD} 25^{+} \mathrm{CD} 127^{\text {low }}$ Foxp $3^{+} \mathrm{T}$ cell levels in children with autoimmune thyroid diseases. Autoimmunity 2013, 46, 222-230.

38. Nocentini, G.; Giunchi, L.; Ronchetti, S.; Krausz, L.T.; Bartoli, A.; Moraca, R.; Migliorati, G.; Riccardi, C. A new member of the tumor necrosis factor/nerve growth factor receptor family inhibits t cell receptor-induced apoptosis. Proc. Natl. Acad. Sci. USA 1997, 94, 6216-6221.

39. Bianchini, R.; Bistoni, O.; Alunno, A.; Petrillo, M.G.; Ronchetti, S.; Sportoletti, P.; Bocci, E.B.; Nocentini, G.; Gerli, R.; Riccardi, C. CD4 ${ }^{+} \mathrm{CD} 25^{\text {low }} \mathrm{GITR}^{+}$cells: A novel human $\mathrm{CD} 4^{+}$T-cell population with regulatory activity. Eur. J. Immunol. 2011, 41, 2269-2278.

40. Alunno, A.; Petrillo, M.G.; Nocentini, G.; Bistoni, O.; Bartoloni, E.; Caterbi, S.; Bianchini, R.; Baldini, C.; Nicoletti, I.; Riccardi, C.; et al. Characterization of a new regulatory $\mathrm{CD}^{+}{ }^{+} \mathrm{T}$ cell subset in primary sjogren's syndrome. Rheumatology (Oxford) 2013, 52, 1387-1396.

41. Nocentini, G.; Alunno, A.; Petrillo, M.; Bistoni, O.; Bartoloni, E.; Caterbi, S.; Ronchetti, S.; Migliorati, G.; Riccardi, C.; Gerli, R. Expansion of regulatory GITR ${ }^{+} \mathrm{CD} 25^{\text {low/- }} \mathrm{CD} 4{ }^{+} \mathrm{T}$ cells in systemic lupus erythematosus patients. Arthritis Res. Ther. 2014, 16, 444.

(C) 2014 by the authors; licensee MDPI, Basel, Switzerland. This article is an open access article distributed under the terms and conditions of the Creative Commons Attribution license (http://creativecommons.org/licenses/by/4.0/). 